\title{
Pentosan Polysulfate A Miss Understood Cancer Terminator - Historical Review and A Future Path Forward Proposal
}

\author{
Jamil Hantash* \\ VJO Biotech Support LLC, USA
}

Submission: March 19, 2018; Published: April 12, 2018

*Corresponding author: Jamil Hantash, PhD, VJO Biotech Support LLC, Bordentown, NJ, USA, Email: vjobiotech@gmail.com

\begin{abstract}
Pentosan polysulfate (PPS, $\beta$-Xylan 2,3-bis(hydrogen sulfate) with a 40 -methyl- $\alpha$-D-glucuronate is a semi-synthetic polysulfated xylan approved for the relief of thrombi and interstitial cystitis in humans and osteoarthritis in dogs and horses. Since 1992 PPS have been studied for effect on defeating cancer. The in vitro data up until recant all showed consistent effectiveness of PPS to control tumor cell growth and even initiating cell death mechanism which makes it a great candidate for a cancer therapeutic agent. However, in vivo studies all showed no statistical significance of PPS in treating cancer. This is mainly as we present in this review due to miss understanding of the chemical and physiochemical characteristics of PPS. It is our believe that the formulation of a conjugated entity of three part can allow PPS to defeat cancer cells. The path forward relies on the ability to further formulate PPS and understand the mechanism of action. That is the main goal of this mini review is to set the light on the path forward and guide scientists to what may become a great solution.
\end{abstract}

Keywords: Pentosan; Tumor; Cell growth; Breast cancer; Prostate cancer

\section{Introduction}

Pentosan polysulfate sodium (PPS) also known as Elmiron was Federal drug and food administration (FDA) approved for the relief of bladder pain or discomfort associated with interstitial cystitis (IC) [1]. It is a non-toxic and non-immunogenic therapeutic agent that could be tolerated at a dose of $400 \mathrm{mg} /$ $\mathrm{m}^{2}$ per day. It is therefore attractive to many scientists and has been studied in the area of oncology extensively since 1992 if not earlier. To understand the true hypothesis behind PPS and use in terminal late stages of cancer we will review the published facts thus far.

The story of PPS with oncology started in 1992 when Zugmaier et al. [2] reported the in vitro findings that made PPS an attractive candidate for late stage cancer treatment in the clinic. It is known that the locally secreted polypeptide growth factors that have autocrine and paracrine functions play a key role in inducing vascularization essential for tumor growth and metastasis. These growth factor can be targeted for tumor therapy. PPS have been shown to block growth subcutaneous human tumor xenografted in nude mice and angiogenesis induced by heparin binding, Kaposi's sarcoma derived fibroblast growth factor K-FG. In 1992 the study by Zugmaier et al. [2] examined the effect of PPS on seven human cell lines established from breast, prostate, epidermal and lung carcinomas and rhabdomyosarcoma. The subcutaneous growth of the tumor from all cell lines in athymic nude mice was inhibited in a dose dependent fashion by daily intraperitioneal injection of PPS. However, six of the seven cell lines showed resistance to PPS in soft agar cloning assay, which indicate that that there is no dependence on autocrine stimulation by heparin binding growth factors. PPS remained a strong candidate to be tested clinically because the study also showed that PPS inhibited the stimulus of heparin growth factors which is related to the cell lines paracrine activity. Pienta et al. [3] reported in 1992 that PPS showed the ability to inhibit the growth of highly metastatic MAT-LyLu (MLL) Dunning R3327 prostate cancer cell line only at toxic doses. In vitro PPS showed a potent inhibition of cell motility. Therefore, by blocking cell motility, PPS becomes a candidate therapeutic agent to be used to inhibit both tumor growth and metastasis [3].

Finally, in 1995 PPS was tested in the clinic. Swain et al. [4] reported after conducting a clinical trial where 19 patients with various adult solid tumors were treated with PPS administered subcutaneous. The data showed that there was an increase in anticoagulant activity as measured by activated partial thromboplastin (aPTT) and there were no objective responses in the patients. This was exciting news that triggered further testing of PPS clinically on oncology terminal or late stage 
patients [4]. Marshall et al. [5] reported in 1997 after conducting a phase I clinical trials on advanced cancer patients that PPS showed no statistically objective response after dosing up to $400 \mathrm{mg} / \mathrm{m}^{2}$. Only three patients had prolonged stabilization of previously progressing disease. The analytical testing showed that there was a marked accumulation of PPS upon chronic administration. Serum and urine bFGF levels failed to show consistent interpretable pattern, however, there was an inverse relationship between PPS and bFGF levels in vivo [5]. These failed clinical trials forced the scientific community to conduct additional in vitro testing to understand the potential of PPS as a therapeutic oncology treatment. Are we back to square one? Did the scientific community miss the fact that PPS does not accumulate when administered orally for IC and that PPS worked in vitro due to its availability at an effective low dose to impact tumor cells and shut down growth mechanism?

In 2003 Elliot et al. [6] reported that PPS has a significant effect on growth and extracellular matrix (ECM) of smooth muscle cells derived from vascular tissues. They showed through in vitro experiments that the administration of PPS decreased proliferation as well as ECM production in prostate smooth muscle. The hypothesis was that, since smooth muscle proliferation and ECM are involved in the pathophysiology of Benign prostate hyperplasia (BPH) then PPS has a therapeutic effect. This was yet again another success in the in vitro experiments supporting that PPS can be used in oncology as a treatment [6]. In 2004 Zaslau et al. [7] reported that in breast cancer cell lines MCF-7, ZR75-1 and HTB26 that were treated with PPS, had no growth stimulatory or inhibitory effects on estrogen independent breast cancer cells in vitro. PPS did display both growth inhibitory and stimulatory effects on estrogen dependent breast cancer cell line by apoptotic mediated mechanism. Therefore, this was considered another success story in the in vitro experiments supporting PPS as a candidate to treat breast cancer. In another in vitro experiment, Zaslau et al. [7] reported in 2006 that PPS significantly inhibited the growth of the androgen dependent LnCaP cell line, the androgen independent PC3 and DU145 cell lines. The reported data showed that PPS exhibited decreased cellular growth in both androgen dependent and independent prostate cancer cells [8]. This also support PPS as a successful in vitro candidate to be tested in vivo for the use to treat late stage cancer patients. In our opinion, the in vitro data since 1992 showed strong evidence that PPS can be used in late stage cancer treatment. In our opinion the lack of understanding of PPS chemically and physiochemical properties miss lead the cells or even initiate cell scientific community clinically to a path the resulted in failure. Let now examine the proposed mechanism of action of PPS on tumor cells. Zugmaier et al. [2] in 1992 proposed that PPS acts by blocking the paracrine effects of heparin binding growth factors released from the tumor cells. Zaslau et al. [7] proposed that PPS display both growth inhibitory and stimulatory effects on estrogen dependent breast cancer cell line by apoptotic mediated mechanism. In 1992 when Zugmaier et al. [2] proposed that PPS can block tumorigenesis in athymic nude mice injected with moderate numbers of tumorigenic human carcinoma or sarcoma cells [2]. PPS can block the paracrine effects of tumor cells but when tumor attain large numbers and size PPS fail to be effective. Thus, it is possible that PPS has therapeutic effect in patients with minimal residual disease such as advanced local breast cancer. It also could have preventative effect. Zaslau et al. [7] in 2006 proposed that reduction of VGEF after administering PPS in vitro experiments is a strong evidence that PPS act by regulating angiogenesis [8].

\section{Discussion}

The main objective of this review is to set the path forward for a new look at PPS as a therapeutic anti tumor agent. The question is how does PPS work?

Our view is that PPS showed since 1992 in in vitro that it is a great successful candidate to treat late stage tumor cells. We believe that the most important fact is that the ECM has a wide range of cellular growth factors stored. In the complex dynamics of tumor invasion and metastasis it involves the destruction of ECM by enzymes such as threonine proteases, serine proteases, and matrix metalloproteinases. It is important to explain and understand the term Durotaxis and its role in cancer. Durotaxis is the migration of cells in the direction of greater stiffness (ECM plays a key role in that process). It is known that tumors are stiffer than surrounding tissue. The stiffness is a result of the involvement of many cell types when forming a tumor (cell types like endothelial cells, fibroblasts for example). It has been shown that mice have demonstrated that tumor cells invade adjacent stroma along the stiff collagen fibers. It was also demonstrated that stiff collagen alignments are used to identify focal breast tumor sites microinvasion [9]. Researchers also shown that the stiffer the tumor, the more increased metastasis and the more decreased survival. Now we believe that PPS must be coupled with a known entity that is essential in fighting tumor growth Vitamin B17. The D-amygdalin form of vitamin B17 helps with regression and growth of cancerous cells and tumors as it exhibits selective killing effect on mutated cells (apoptosis). It also stimulates the immune system by causing a statistically significant increase in the ability of a patient's white blood cells to attack harmful cells. In our opinion the future in a platform where PPS is conjugated to vitamin B17 and a fusion protein. The fusion protein can be selected from libraries that contain proteins which bind specific, and with high affinity to cell receptors over expressed in tumor cells. We believe such platform will allow the delivery of an effective PPS at a low dose to the tumor cells at a therapeutic dose to stop growth of tumath mechanism. One must remember from the history review in this mini review that PPS work in in vitro but fails in in vivo due to inability to get into cells at a therapeutic level and without the support of vitamin B17 that is essential for cell death. 


\section{Conclusion}

Since 1992 researchers showed that in vitro PPS is effective in treating tumor cells. However, in in vivo all clinical studies showed that PPS has no statistical significant in the treatment of cancer. We presented our hypothesis and believe for the path forward. PPS coupled with fusion protein to penetrate and target tumor cells along with a conjugated vitamin B17 could be the answer to the failed PPS acting in in vivo.

\section{References}

1. Ghosh P, Smith M (2002) Osteoarthritis, genetic and molecular mechanisms. Biogerontology 3 (1-2): 85-88.

2. Zugmaier G, Lippman ME, Wellstein A (1992) Inhibition by Pentosan Polysulfate (PPS) of heparin-binding growth factors released from tumor cells and blockage by PPS of tumor growth in animals. J Nat Cancer Inst 84(22): 1716-1724.

3. Pienta KJ, Murphy BC, Isaacs WB, Isaacs JT, Coffey DS (1992) Effect of pentosane, a novel cancer chemotherapeutic agent, on prostate cancer cell growth and motility. Prostate 20(3): 233-241.
4. Swain SM, Parker B, Wellstein A, Lippman ME, Steakley C, et al. (1995) Phase I trial of pentosane polysulfate. Invest New Drugs 13(1): 55-62.

5. Marshall JL, Wellstein A, Rae J, DeLap RJ, Phipps K, et al. (1997) Phase I trial of orally administered pentosane polysulfate in patients with advanced cancer. Clin Cancer Res 3(12 Pt 1): 2347-2354.

6. Elliot SJ, Zorn BH, McLeod DG, Moul JW, Nyberg L, et al. (2003) Pentosan polysulfate decreases prostate smooth muscle proliferation and extracellular matrix turnover. Prostate Cancer Prostatic Dis 6(2): 138-142.

7. Zaslau S, Riggs DR, Jackson BJ, Adkins FC, John CC, et al. (2004) In vitro effect of pentosane polysulfate against malignant breast cells. Am J Surg 188(5): 589-592.

8. Zaslau S, Sparks S, Riggs D, Jackson B, Kandzari SJ (2006) Pentosan polysulfate (Elmiron): in vitro on prostate cancer cells regarding cell growth and vascular endothelial growth factor production. Am J Surg 192(5): 640-643.

9. Mesquita FS, Dyer SN, Heinrich DA, Bulun SE, Marsh EE, et al. (2010) Reactive oxygen species mediate mitogenic growth factor signaling pathways in human leiomyoma smooth muscle cells. Biol Reprod 82(2): 341-351.

\section{Your next submission with Juniper Publishers} will reach you the below assets

- Quality Editorial service

- Swift Peer Review

- Reprints availability

- E-prints Service

- Manuscript Podcast for convenient understanding

- Global attainment for your research

- Manuscript accessibility in different formats

( Pdf, E-pub, Full Text, Audio)

- Unceasing customer service

Track the below URL for one-step submission https://juniperpublishers.com/online-submission.php 